\title{
Automated Counting and Identification of Cell Colonies Based on Distance Transform and Progressive Erosion
}

\author{
Zhifeng Hu \\ School of Electrical Engineering, Suzhou Vocational University, Suzhou, China \\ huzf@jssvc.edu.cn
}

\begin{abstract}
In this paper, a novel algorithm is proposed to facilitate the automated counting and identification of cell colonies in a Petri dish. In order to detect all the individual colonies, the original Petri dish image is converted to a black white version, after which a distance transform is carried out to highlight the colony centers. Finally, a progressive erosion approach is adopted to isolate and identify all the individual colonies. Experiment results show that the algorithm performs well on practical Petri dish images.
\end{abstract}

Index Terms - Digital Image Processing, Automated Colony Identification, Distance Transform.

\section{I . Introduction}

The cell colony formation method is a standard procedure to determine the growth rate of bacterial cells in biological experiments. In a typical setting, a certain number of cells are plated into an agar plate where some nutrient has been randomly distributed. After some time, the cells that happen to be in suitable locations would reproduce and give rise to colonies. Naturally, the number and distribution of the colonies on the plate provide important measurements for determining the growth rate of the cells. Traditionally, this is done through manually marking each colony that appear on the plate. This task can be eyestraining, tedious and time consuming. The results thus obtained also fail to be consistent due to human errors. Therefore, in recent years, algorithms based on computer vision have been proposed to complete this task automatically [1], [2]. Hough transform was employed to effectively count the single colonies[3]. As an alternative, the authors in [4] employed an algorithm based on forward scattering to identify the bacterial colonies. In [5], top hat filtering was utilized followed by a segmentation algorithm to successfully count the bacterial colonies on agar plates. However, many of these algorithms result in high false positive and true negative rates. There remains much room for performance improvement.

In this paper, a novel approach is proposed for the problem of automated counting of cell colonies. The progressive erosion based algorithm was specifically designed to address key tech- nical issue such as nonuniform lighting, overlapping colonies and the interference from the plate background. Experiment results show that the algorithm yield satisfactory results.

\section{II . Program overview}

In this section, a brief account of the overall system design is given. Detailed design in the various stages of the algorithm will be described in the following sections. The goal of the program is to count and mark all the individual colonies in a petri dish. Given a picture as in Fig. 1, the algorithm aims to automatically mark all the colonies contained within the plate. The main difficulty of such a task lies in the presence of the many connected colonies as shown in Fig. 1. When several colonies are so close as to be connected to each other, it is important to separate them before accurately locating them individually and obtaining an accurate estimate of their total number. Several specialized image processing procedure have been adopted in this paper to tackle with this matter. The overall block diagram of the algorithm is shown in Fig. 2.

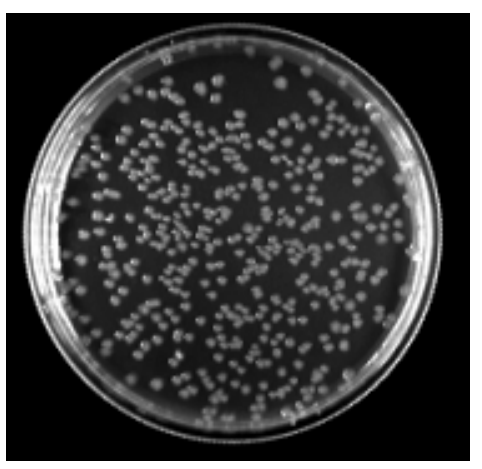

Figure 1. Petri dish image

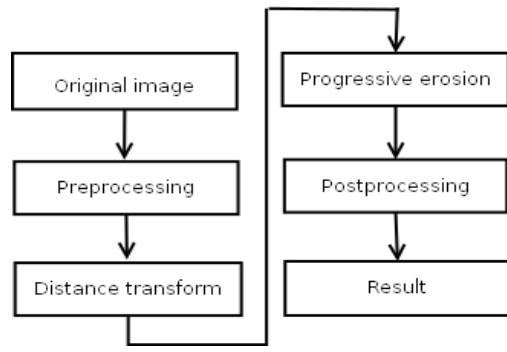

Figure 2. Block diagram of the algorithm

\footnotetext{
* This research is supported in part by Suzhou Vocational University through the grant 2011SZDQ07.
} 


\section{III . Preprocessing}

Upon obtaining the original color image of a petri dish as shown in Fig. 1, the first step in the algorithm is to convert it to a gray level image. Next, a global threshold value must be determined to convert the image to a black white image. Instead of adopting a constant global threshold value, an adaptive procedure for computing the threshold value based on the input gray level image is adopted in this paper. The resulting performance is robust to the variations in the illumination level of the input image. To compute the optimal threshold value, let Ig denote gray level image, and let $\mathrm{x}$ denote the pixel intensity values of Ig. The optimal threshold value $u$ can be computed as follows:

$$
\begin{aligned}
& u^{*}=\min \sum_{x>u}\left(x-c_{1}\right)^{2}+\sum_{x \leq u}\left(x-c_{2}\right)^{2} \\
& c_{1}=\frac{\sum_{x>u} x}{\operatorname{card}(\{x \mid x>u\})}, c_{2}=\frac{\sum_{x \leq u} x}{\operatorname{card}(\{x \mid x \leq u\})}
\end{aligned}
$$

Where $c_{1}$ stands for the mean intensity value of the pixels whose intensity is greater than $u$, and $c_{2}$ stands for the mean intensity value of the pixels whose intensity is less than $u$. In effect, the above equation computes the optimal threshold value such that the intra-class variance of the gray level intensity is minimized. Therefore, a conversion to a black white image according to the computed threshold value would render most of the colony pixels white while leaving the rest of the pixels black. The resulting black white image is shown in Fig. 3. The next procedure is to locate the dish itself so as to target the recognition part of the algorithm on the area inside the dish. In order to achieve this, notice that the dish appears as a very large connected component in Fig. 3. Therefore, all the 4-way connected components in the black white image Ibw can be computed and the dish itself can be identified as the largest one among them by pixel count. The image of the dish thus extracted is shown in Fig.4. A bounding box of this connected component can then be computed, which would then yield the size as well as the center of the dish. At this moment, all the pixels outside the dish can be set to black, and the dish itself can be subtracted from the black white image. As a result, the only remaining white pixels correspond to the original colonies inside the dish, which make the target for the recognition part of the algorithm in the next section.

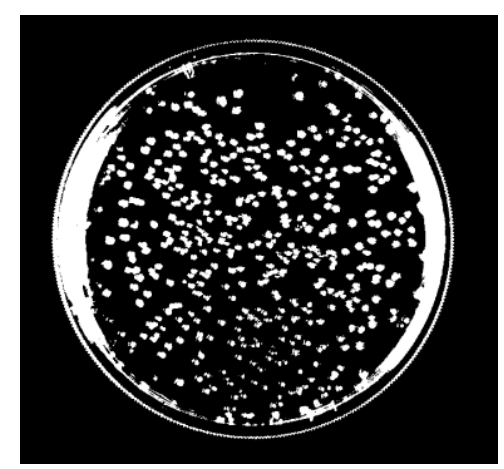

Figure 3. Black white image

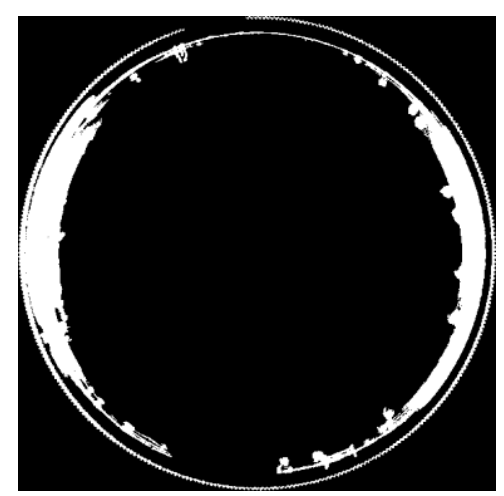

Figure 4. Dish located as the largest connected component

\section{IV . Recognition Of Colonies Via Progressive Erosion}

Once preprocessing is completed, it is easy to compute the number and location of the clusters within the dish. Here a cluster is defined as a group of colonies that are connected together in the black white image. A typical cluster is shown in Fig. 5. The next step is crucial to the overall performance of the algorithm: it is necessary to separate out all the individual colonies in the cluster and to identify their location. As shown in Fig. 5, although the location and number of colonies are apparent to the human eye, it is impossible to identify them separately based solely on a measure of pixel connectedness. Therefore, a distance transform is defined in this section tofa- cilitate a progressive erosion scheme that is aimed at filtering out the individual colonies in the cluster.

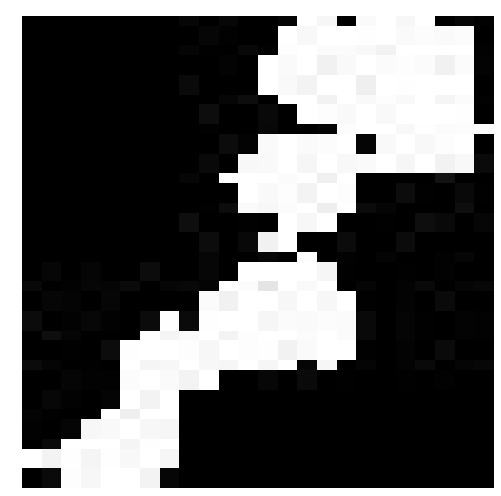

Figure 5. A typical colony cluster 


\section{A . Distance Transform}

A distance transform is a mapping from all the pixel locations in the cluster to a real valued function. In this paper, the distance transform is defined as follows: for each point within the cluster, its distance function value is defined as the distance from itself to its closest black point in its neighborhood. The intuition here is that colony centers would be assigned large values while peripheral pixels of a colony would have smaller values assigned to them, even though they are connected to a nearby colony. Mathematically, the distance transform can be expressed as: where $(x, y)$ stands for a pixel position in the cluster and Ibw stands for the black white image. As a result, colony centers would have locally maximum values, and peripheral pixels would have small values. If the result of the distance transform is scaled to a range of $[0,255]$, this effect can be visualized with an equivalent grayscale image as shown in Fig. 6, where the grayscale level of a pixel is proportional to its distance function value.

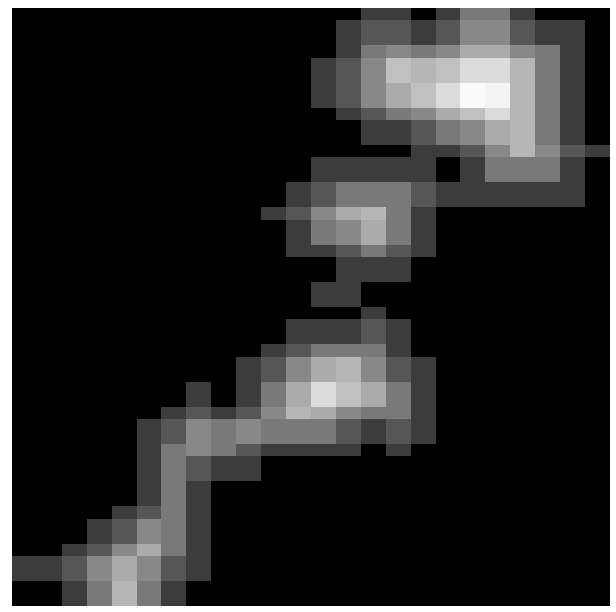

Figure 6. Distance transform result

\section{B. Progressive Erosion}

The next step is to carry out progressive erosion to extract the individual colonies inside a cluster. In order to do this, progressive graylevel thresholding can be applied on the dis- tance transform image. While increasing the threshold value, connected components are shrinking and splitting, resulting in individual colonies being separated out. Throughout this process, the number and locations of the individual colonies can be recorded. The progressive erosion process can terminate when all the remaining colonies in the cluster drop below a predetermined threshold. The intermediate results are shown in Fig. 7.

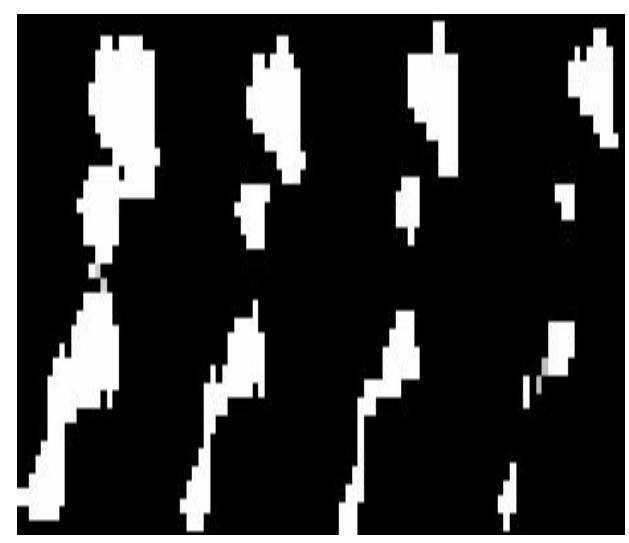

Figure 7. From left to right: intermediate results of progressive erosion on the cluster shown in Fig.5

\section{Postprocessing}

The distance transform and progressive erosion can be ap- plied to all the clusters in the black white image as shown in Fig. 5, so that all the individual colonies can be identified and recorded. Once all the clusters have been processed, the locations of all the detected colonies can be marked on the original image to visualize the detection result. The final result is as shown in Fig. 8.

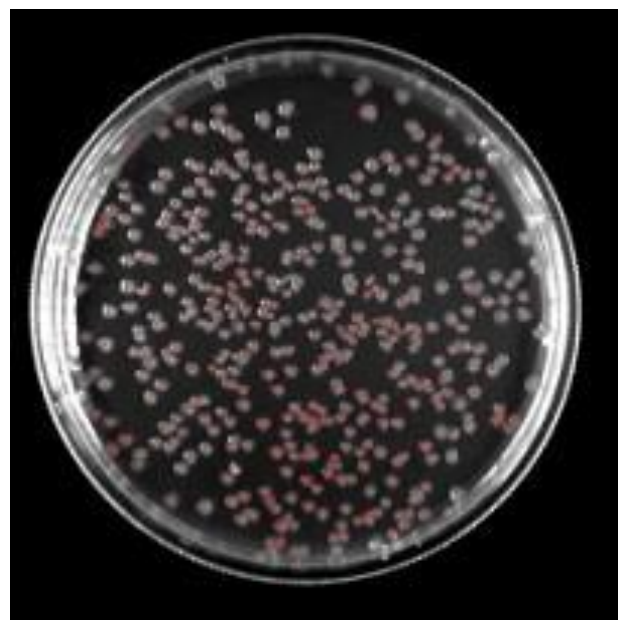

Figure 8. Final result

\section{VI . Experiments}

To verify and test the performance of the algorithm, it has been applied to a group of ten Petri dish images. Some of the results are shown in Fig. 9. As can be seen from the result images, the algorithm performs very well on the test images. 


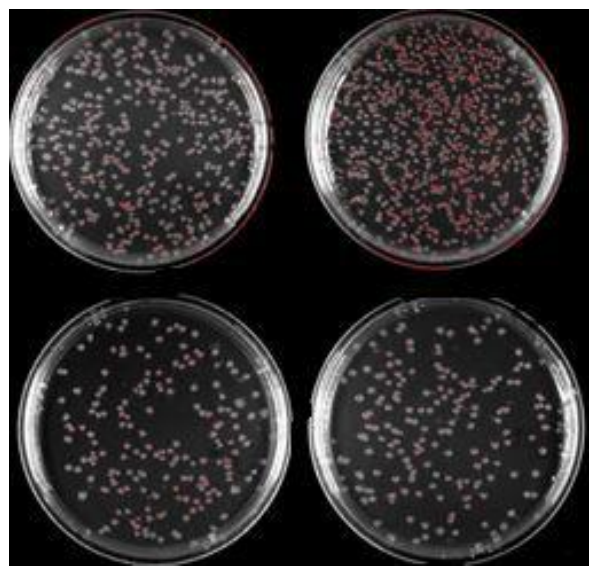

Figure 9. Experiment result

\section{VII . Conclusion}

In this paper, a novel algorithm is proposed to facilitate the automated counting and identification of cell colonies in a Petri dish. The original Petri dish image is firstly converted to a black white version. A distance transform is then carried out to high- light the colony centers. Finally, a progressive erosion approach is adopted to isolate and identify all the individual colonies. Experiment results show that the algorithm performs well on practical Petri dish images. In the future, more sophisticated models of the colony clusters can be built and incorporated into the algorithm to further enhance its performance.

\section{References}

[1] D.P. Mukherjee, A.Pal, S.E. Sarma, and D.D. Majumder, "Bacterial colony counting using distance transform," International Journal of Bio-Medical Computing, vol.38, pp. 131-140, 1995.

[2] G.Corkidi, R.Diaz-Uribe, J.L. Folch-Mallol, and J.Nieto-Sotelo, "COVASIAM: an image analysis method that allows detection of confluent microbial colonies and colonies of various sizes for automated counting." Applied and environmental microbiology, vol.64, no.4, pp. 1400-4, Apr. 1998.

[3] P.R. Barber, B.Vojnovic, J.Kelly, C.R. Mayes, P.Boulton, M.Woodcock, and M.C. Joiner, "An Automated Colony Counter Utilising A Compact Hough Transform," Medical Image Understanding and Analysis, pp. 2-5, 2000.

[4] E.Bae, A.Aroonnual, A.K. Bhunia, J.P. Robinson, and E.D. Hirleman, "System automation for a bacterial colony detection and identification instrument via forward scattering," Measurement Science and Technology, vol.20, no.1, p. 015802, Jan. 2009.

[5] S.D. Brugger, C.Baumberger, M.Jost, W.Jenni, U.Brugger, and K.Mühlemann, "Automated counting of bacterial colony forming units on agar plates." PloS one, vol.7, no.3, p. e33695, Jan. 2012. 